\title{
Malnutrition, renal dysfunction and left ventricular hypertrophy synergistically increase the long-term incidence of cardiovascular events
}

\author{
Keisuke Maruyama ${ }^{1}$, Naoki Nakagawa ${ }^{1}$, Erika Saito ${ }^{1}$, Motoki Matsuki ${ }^{1}$, Naofumi Takehara ${ }^{1}$, Kazumi Akasaka ${ }^{2}$, \\ Nobuyuki Sato ${ }^{1}$ and Naoyuki Hasebe ${ }^{1}$
}

Although malnutrition indicates an unfavorable prognosis in some clinical settings, the synergistic impact of nutritional state, renal dysfunction and left ventricular hypertrophy (LVH) on cardiovascular events is unknown. Among 338 patients aged 40-80 years who underwent echocardiographic evaluation between 2003 and 2005, 161 patients who were followed for $>7$ years were recruited. Malnutrition was defined as a geriatric nutritional risk index (GNRI) of $\leqslant 96$. The mean patient age was $63.5 \pm 9.2$ years; the mean estimated glomerular filtration rate (eGFR) was $72.9 \pm 18.7 \mathrm{ml} \mathrm{min}^{-1}$ per $1.73 \mathrm{~m}^{2}$; the mean LV mass index was $114 \pm 33 \mathrm{~g} \mathrm{~m}^{-2}$; and the mean GNRI was $100.4 \pm 6.0$. Among the patients, $25 \%(n=40)$ had an eGFR of $<60 \mathrm{ml} \mathrm{min}^{-1}$ per $1.73 \mathrm{~m}^{2}, 29 \%(n=46)$ exhibited chronic kidney disease (CKD) and 37\% $(n=59)$ had LVH. During the follow-up period (median: 96 months), cardiovascular events were observed in 15 patients (9\%). Kaplan-Meier curves showed a significantly higher incidence of cardiovascular events in patients with an eGFR of $<60 \mathrm{ml} \mathrm{min}-1$ per $1.73 \mathrm{~m}^{2}$ (log-rank $P=0.007$ ), a GNRI of $\leqslant 96(P=0.003)$ or LVH $(P=0.010)$. In a Cox regression analysis, eGFR, LVH and GNRI were independent determinants of cardiovascular event incidence after adjusting for age, gender and the presence of hypertension and diabetes. Furthermore, the combination of LVH and lower GNRI was significantly associated with a higher rate of cardiovascular events not only in all patients but also in patients with CKD. In conclusion, malnutrition, low eGFR and LVH were independent determinants of cardiovascular event incidence; they synergistically increased rates of these events in the long term. The evaluation and management of LVH progression and the improvement of nutritional status are critical for preventing cardiovascular complications even in non-dialysis patients.

Hypertension Research (2016) 39, 633-639; doi:10.1038/hr.2016.47; published online 12 May 2016

Keywords: cardiovascular diseases; left ventricular hypertrophy; nutritional status; renal function

\section{INTRODUCTION}

Chronic kidney disease (CKD) is one of the most important risk factors for cardiovascular events worldwide. ${ }^{1,2}$ Left ventricular hypertrophy (LVH) is also common and is a strong predictor of cardiovascular events in patients with $\mathrm{CKD} .{ }^{3,4} \mathrm{LVH}$ can be preventable or even reversible by controlling blood pressure and volume in patients with CKD. ${ }^{5,6}$ Malnutrition has been identified as an independent risk factor for cardiovascular events in dialysis patients. ${ }^{7}$ A recent study revealed higher all-cause (including cardiovascular diseases) mortality rates in predialysis patients with $\mathrm{CKD}$ and malnutrition. ${ }^{8}$ The geriatric nutritional risk index (GNRI) is a simple, well-established nutritional assessment tool for both elderly individuals $^{9}$ and hemo- and peritoneal dialysis patients. ${ }^{10,11}$ It is based on three objective parameters: body weight, height and serum albumin level. We recently reported that the cutoff value of GNRI for predicting all-cause and cardiovascular mortality is 96 in hemodialysis patients. ${ }^{12}$ However, this assessment has not yet been applied to nondialysis patients.

Malnutrition and LVH have already been shown to be associated with cardiovascular events in patients with $\mathrm{CKD}$, but the synergistic impact of malnutrition, low estimated glomerular filtration rate (eGFR) and LVH on cardiovascular events during long-term followup is still unknown. Therefore, we investigated the associations between GNRI, eGFR, LVH and cardiovascular events during a follow-up period of $>7$ years.

\section{METHODS}

Participants

We enrolled 338 patients who were 40 to 80 years of age and who underwent echocardiographic evaluation between June 2003 and May 2005. After

\footnotetext{
${ }^{1}$ Division of Cardiology, Nephrology, Pulmonology and Neurology, Department of Internal Medicine, Asahikawa Medical University, Asahikawa, Japan and ${ }^{2}$ Department of Medical Laboratory and Blood Center, Asahikawa Medical University Hospital, Asahikawa, Japan

Correspondence: Dr N Nakagawa, Division of Cardiology, Nephrology, Pulmonology and Neurology, Department of Internal Medicine, Asahikawa Medical University, Midorigaoka-higashi 2-1-1-1, Asahikawa, Japan.

E-mail: naka-nao@asahikawa-med.ac.jp

Received 3 January 2016; revised 22 February 2016; accepted 4 March 2016; published online 12 May 2016
} 
excluding patients who were on hemodialysis, patients with malignancies or infectious diseases, and patients who were followed for $<7$ years, 161 patients (male, $n=82$; female, $n=79$; mean age, $63.5 \pm 9.2$ years) were enrolled. Age, gender, lipid parameters and conventional cardiovascular risk factors were recorded. This study was performed in strict accordance with the ethical guidelines of the Declaration of Helsinki and was approved by the Ethical Scientific Committee of Asahikawa Medical University. All study participants provided written informed consent.

\section{Data collection}

Body mass index was calculated by dividing weight by height squared $\left(\mathrm{m}^{2}\right)$. We calculated GNRI using serum albumin values, weight and ideal body weight. GNRI was calculated as reported by Yamada et al. ${ }^{10}$ GNRI $=(14.89 \times$ albumin $\left.\left(\mathrm{g} \mathrm{dl}^{-1}\right)\right)+(41.7 \times($ weight/ideal body weight $))$. Note that body weight/ideal body weight was $>1$ when a subject's body weight exceeded their ideal body weight. Ideal body weight was calculated using height and a body mass index of 22 , which is reportedly associated with the lowest morbidity rate in the Asian population. ${ }^{13}$ Hypertension was defined as systolic blood pressure $\geqslant 140 \mathrm{~mm} \mathrm{Hg}$ and/or diastolic blood pressure $\geqslant 90 \mathrm{~mm} \mathrm{Hg}$ or current use of antihypertensive agents. Diabetes was defined as one of the following: fasting blood sugar $\geqslant 126 \mathrm{mg} \mathrm{dl}^{-1}$, non-fasting blood sugar $\geqslant 200 \mathrm{mg} \mathrm{dl}^{-1}$, glycosylated hemoglobin $\geqslant 6.5 \%$ or current use of insulin or oral hypoglycemic agents. Dyslipidemia was defined as a total cholesterol level of $\geqslant 220 \mathrm{mg} \mathrm{dl}^{-1}$, high-density lipoprotein cholesterol level of $<40 \mathrm{mg} \mathrm{dl}^{-1}$ for men and $<50 \mathrm{mg} \mathrm{dl}^{-1}$ for women, triglyceride level of $\geqslant 150 \mathrm{mg} \mathrm{dl}^{-1}$ or treatment with antihyperlipidemic agents. Proteinuria was defined as $1+$ or more on dipstick test.

\section{Echocardiographic evaluation}

Standard echocardiographic measurements were obtained from the left parasternal and apical views according to the recommendations of the American Society of Echocardiography ${ }^{14}$ using an ALOKA alpha 10 ultrasound system (Aloka, Mitaka, Tokyo, Japan). The LV end-diastolic diameter, LV end-systolic diameter, left atrial diameter (LAD), interventricular septum thickness and posterior wall thickness were determined using standard echocardiographic two-dimensional measurements. LV mass (LVM) was calculated with the two-dimensional area-length formula. LVMs were indexed to the body surface area as the LVM index (LVMI). LVH was defined as an LVMI $\geqslant 125 \mathrm{~g} \mathrm{~m}^{-2}$ for men and $\geqslant 110 \mathrm{~g} \mathrm{~m}^{-2}$ for women. ${ }^{15}$ The ejection fraction was calculated using the modified Simpson method. Transmitral inflow was recorded with pulsed wave Doppler recordings at the mitral valve leaflet tips in the apical threechamber view. The peak early diastolic filling velocity $(E)$, peak late diastolic filling velocity $(A), E / A$ ratio and deceleration time of the $E$ wave were measured. The tissuez Doppler imaging program was set to the pulsed wave Doppler mode, and sample volumes were positioned at the septal corner of the mitral annulus. ${ }^{16}$ We then measured the early diastolic mitral inflow velocity and calculated the ratio of $E$ over $e^{\prime}\left(E / e^{\prime}\right)$ to represent LV filling pressure. ${ }^{17}$

\section{Follow-up}

Follow-up data were retrieved from clinical records and/or death certificates by personnel blinded to the anthropometric, body composition and laboratory assessments. Heart failure was defined upon admission as a result of new or worsening heart failure with a New York Heart Association class $\geqslant$ II. Followup began on the date of enrollment and finished at death from any cause or until 31 March 2014, whichever came first.

\section{Statistical analysis}

The results are expressed as the mean \pm s.d. Baseline characteristics between the cardiovascular event group and the cardiovascular event-free group were compared using the $\chi^{2}$ test and the Mann-Whitney $U$-test as appropriate. The relationships among eGFR, echocardiographic function and malnutrition at the time of enrollment were analyzed via univariate linear regression analysis. Uni- and multivariate linear regression analyses and Cox proportional hazards models were performed to examine correlations and independent variables for cardiovascular events. The impact of GNRI, eGFR and LVH on cardiovascular events, including acute coronary syndrome, heart failure, stroke, aortic dissection and aortic rupture were analyzed using Kaplan-Meier curves and log-rank tests. $P$-values $<0.05$ were considered statistically significant. All data were analyzed using SPSS ver. 19.0 Windows (SPSS, Chicago, IL, USA).

\section{RESULTS}

\section{Clinical characteristics}

Table 1 shows the baseline characteristics of all patients during a median follow-up period of $96.0 \pm 15.6$ months. The mean age of the 161 patients at baseline was $63.5 \pm 9.2$ years, $51 \%$ of patients were male, $68 \%$ had hypertension, $24 \%$ had diabetes and $45 \%$ had dyslipidemia. Mean eGFR was $72.9 \pm 18.7 \mathrm{ml} \mathrm{min}^{-1}$ per $1.73 \mathrm{~m}^{2}$. Mean serum albumin level was $4.0 \pm 0.3 \mathrm{mg} \mathrm{dl}^{-1}$ and mean GNRI was $100.4 \pm 6.0$. Among the patients, $25 \%(n=4)$ exhibited an eGFR of $<60 \mathrm{ml} \mathrm{min}{ }^{-1}$ per $1.73 \mathrm{~m}^{2}$ (G3a, $n=29$; G3b, $n=9$; and $\mathrm{G} 4$, $n=2), 9 \%(n=15)$ had proteinuria, 29\% $(n=46)$ were diagnosed with CKD and $37 \%(n=59)$ had LVH. Patients with nephrotic syndrome were not included.

During the follow-up period, 15 (9.3\%) patients experienced cardiovascular events (acute coronary syndrome, $n=7$; heart failure, $n=4$; stroke, $n=2$; aortic dissection, $n=1$; aortic rupture, $n=1$ ). Gender, body mass index, hemoglobin level, and the prevalence of hypertension, diabetes and dyslipidemia were not significantly different between patients who did or did not experience cardiovascular events. Patients who developed cardiovascular events were significantly older, had a lower eGFR, serum albumin levels, $e^{\prime}$ and GNRI, and had a higher LVMI. The use of diuretics, angiotensin II receptor blockers and angiotensin-converting enzyme inhibitors was significantly higher in patients who developed cardiovascular events.

\section{Correlations between eGFR, LVMI and GNRI}

Univariate correlations between eGFR, LVMI, GNRI and various parameters at baseline are listed in Table 2. eGFR correlated significantly and negatively with age, the prevalence of proteinuria, $\mathrm{LAD}$ and LVMI, and positively with $e^{\prime}$, serum albumin levels and GNRI in univariate linear regression analysis. LVMI correlated significantly and negatively with eGFR and $e^{\prime}$, and it correlated significantly and positively with proteinuria, $\mathrm{LAD}$ and $E / e^{\prime}$. In contrast, GNRI correlated significantly and positively with body mass index, hemoglobin level, serum albumin concentration and eGFR. GNRI did not correlate with LAD, LVMI and LV end-diastolic diameter, suggesting that GNRI occurred irrespective of volume overload.

\section{Follow-up data}

Univariate correlations between the incidence of cardiovascular events and various parameters are listed in Table 3. Cardiovascular events were significantly and positively associated with age, the presence of proteinuria, LAD, E/e $e^{\prime}$ and LVMI; cardiovascular events were negatively correlated with eGFR, serum albumin level and GNRI in univariate linear regression analysis. Next, we analyzed independent determinants of cardiovascular events using multivariate linear regression analyses, the results of which are presented in Table 4. LVMI was positively associated, whereas eGFR and GNRI level were negatively associated with cardiovascular events. Furthermore, we investigated the independent variables for cardiovascular events using Cox proportional hazard models. Age (hazard ratio 1.121, 95\% confidence interval (CI) 1.013-1.241), eGFR (hazard ratio 0.961, 95\% CI 0.928-0.995), GNRI (hazard ratio 0.886, 95\% CI 0.807-0.973) and LVMI (hazard ratio $1.017,95 \%$ CI 1.002-1.033) were significantly associated with the occurrence of cardiovascular events after adjusting 
Table 1 Baseline characteristics of the study patients

\begin{tabular}{|c|c|c|c|c|}
\hline & Total & CV events (+) & CV events (-) & P-value \\
\hline Number & 161 & 15 & 146 & - \\
\hline Age (year) & $63.5 \pm 9.2$ & $68.4 \pm 6.4$ & $63.0 \pm 9.3$ & 0.036 \\
\hline Gender, male & $82(51 \%)$ & $10(67 \%)$ & $72(49 \%)$ & 0.202 \\
\hline Body mass index $\left(\mathrm{kg} \mathrm{m}^{-2}\right)$ & $23.9 \pm 3.6$ & $22.3 \pm 2.8$ & $24.1 \pm 3.6$ & 0.073 \\
\hline Systolic blood pressure (mm Hg) & $130.8 \pm 20.1$ & $138.0 \pm 28.7$ & $130.1 \pm 19.1$ & 0.144 \\
\hline Diastolic blood pressure (mm Hg) & $76.7 \pm 12.0$ & $77.6 \pm 13.3$ & $76.6 \pm 11.9$ & 0.775 \\
\hline Heart rate (beat per minute) & $69.1 \pm 10.8$ & $67.1 \pm 10.4$ & $69.3 \pm 10.8$ & 0.504 \\
\hline eGFR (ml min ${ }^{-1}$ per $\left.1.73 \mathrm{~m}^{2}\right)$ & $72.9 \pm 18.7$ & $56.4 \pm 20.2$ & $74.6 \pm 17.7$ & 0.002 \\
\hline Proteinuria (yes $=1$, no $=0$ ) & $15(9 \%)$ & $4(27 \%)$ & $11(8 \%)$ & 0.016 \\
\hline Hemoglobin $\left(\mathrm{g} \mathrm{dl}^{-1}\right)$ & $13.4 \pm 1.6$ & $12.9 \pm 2.3$ & $13.4 \pm 1.5$ & 0.300 \\
\hline Serum albumin ( $\mathrm{g} \mathrm{dl}^{-1}$ ) & $4.0 \pm 0.3$ & $3.7 \pm 0.4$ & $4.0 \pm 0.3$ & 0.006 \\
\hline GNRI & $100.4 \pm 6.0$ & $95.1 \pm 8.2$ & $100.9 \pm 5.5$ & 0.004 \\
\hline Hypertension & $109(68 \%)$ & $13(87 \%)$ & $96(66 \%)$ & 0.100 \\
\hline Diabetes & $39(24 \%)$ & $4(27 \%)$ & $35(24 \%)$ & 0.817 \\
\hline Dyslipidemia & $72(45 \%)$ & $9(60 \%)$ & $63(43 \%)$ & 0.213 \\
\hline \multicolumn{5}{|l|}{ Antihypertensive agents } \\
\hline Angiotensin II receptor blockers & $66(41 \%)$ & $10(67 \%)$ & $56(38 \%)$ & 0.034 \\
\hline Angiotensin-converting enzyme inhibitors & $18(11 \%)$ & $4(27 \%)$ & $14(10 \%)$ & 0.046 \\
\hline Calcium channel blockers & $69(43 \%)$ & $7(47 \%)$ & $62(42 \%)$ & 0.755 \\
\hline$\beta$-Blockers & $44(27 \%)$ & $6(40 \%)$ & $38(26 \%)$ & 0.249 \\
\hline Diuretics & $24(15 \%)$ & $8(53 \%)$ & $16(11 \%)$ & $<0.001$ \\
\hline Statins & $60(37 \%)$ & $9(60 \%)$ & $51(35 \%)$ & 0.057 \\
\hline \multicolumn{5}{|l|}{ Echocardiographic parameters } \\
\hline LV end-diastolic diameter (mm) & $47.9 \pm 5.0$ & $49.3 \pm 6.8$ & $47.7 \pm 4.8$ & 0.482 \\
\hline LV end-systolic diameter (mm) & $29.3 \pm 5.5$ & $30.5 \pm 7.2$ & $29.2 \pm 5.3$ & 0.900 \\
\hline Interventricular septum thickness (mm) & $9.8 \pm 1.9$ & $11.5 \pm 2.8$ & $9.6 \pm 1.7$ & 0.005 \\
\hline Posterior wall thickness (mm) & $9.0 \pm 1.4$ & $9.9 \pm 1.7$ & $8.9 \pm 1.3$ & 0.027 \\
\hline $\mathrm{LAD}(\mathrm{mm})$ & $38.0 \pm 5.5$ & $41.3 \pm 5.6$ & $37.7 \pm 5.4$ & 0.001 \\
\hline LV ejection fraction (\%) & $71.1 \pm 7.3$ & $69.5 \pm 9.6$ & $71.2 \pm 7.0$ & 0.516 \\
\hline$E / e^{\prime}$ & $6.5 \pm 3.2$ & $8.5 \pm 5.3$ & $6.3 \pm 2.9$ & 0.206 \\
\hline$e^{\prime}\left(\mathrm{cm} \mathrm{s}^{-1}\right)$ & $10.4 \pm 5.0$ & $8.4 \pm 4.4$ & $10.6 \pm 5.0$ & 0.039 \\
\hline LVMI ( $\left.\mathrm{g} \mathrm{m}^{-2}\right)$ & $113.9 \pm 32.6$ & $147.4 \pm 43.7$ & $110.5 \pm 29.3$ & 0.001 \\
\hline
\end{tabular}

Variables are presented as mean \pm s.d., or number (percentage).

Abbreviations: CV, cardiovascular; $e^{\prime}$, early diastolic mitral annulus motion; $E$, early mitral inflow; eGFR, estimated glomerular filtration rate; GNRI, geriatric nutritional risk index; LAD, left atrial diameter; LV, left ventricular; LVMI, left ventricular mass index.

Table 2 Univariate regression analyses of eGFR, LVMI and GNRI

\begin{tabular}{|c|c|c|c|c|c|c|}
\hline \multirow[b]{2}{*}{ Variable } & \multicolumn{2}{|c|}{ eGFR } & \multicolumn{2}{|c|}{ LVMI } & \multicolumn{2}{|c|}{ GNRI } \\
\hline & r & P-value & $r$ & $\mathrm{P}$-value & r & P-value \\
\hline Age & -0.198 & 0.012 & 0.173 & 0.029 & 0.036 & 0.648 \\
\hline Gender $(m=1, f=0)$ & -0.064 & 0.422 & 0.288 & $<0.001$ & 0.017 & 0.834 \\
\hline Body mass index & -0.023 & 0.771 & 0.026 & 0.741 & 0.450 & $<0.001$ \\
\hline Systolic blood pressure & -0.039 & 0.623 & 0.177 & 0.025 & 0.120 & 0.131 \\
\hline Diastolic blood pressure & 0.080 & 0.315 & 0.070 & 0.381 & 0.141 & 0.075 \\
\hline Heart rate & 0.038 & 0.640 & -0.125 & 0.124 & -0.020 & 0.806 \\
\hline eGFR & - & - & -0.272 & $<0.001$ & 0.172 & 0.029 \\
\hline Proteinuria (yes $=1$, no $=0$ ) & -0.303 & $<0.001$ & 0.277 & $<0.001$ & -0.082 & 0.299 \\
\hline Hemoglobin & 0.139 & 0.078 & 0.057 & 0.473 & 0.466 & $<0.001$ \\
\hline Serum albumin & 0.200 & 0.011 & -0.125 & 0.113 & 0.932 & $<0.001$ \\
\hline GNRI & 0.172 & 0.029 & -0.127 & 0.109 & - & - \\
\hline LV end-diastolic diameter & -0.076 & 0.339 & 0.517 & $<0.001$ & 0.052 & 0.516 \\
\hline LAD & -0.271 & 0.001 & 0.499 & $<0.001$ & 0.142 & 0.073 \\
\hline LV ejection fraction & 0.028 & 0.727 & -0.159 & 0.045 & -0.005 & 0.947 \\
\hline$E / e^{\prime}$ & -0.128 & 0.107 & 0.244 & 0.002 & -0.002 & 0.979 \\
\hline$e^{\prime}$ & 0.188 & 0.017 & -0.298 & $<0.001$ & -0.083 & 0.296 \\
\hline LVMI & -0.272 & $<0.001$ & - & - & -0.127 & 0.109 \\
\hline
\end{tabular}

Abbreviations: $e^{\prime}$, early diastolic mitral annulus motion, $E$, early mitral inflow; eGFR, estimated glomerular filtration rate; GNRI, geriatric nutritional risk index; LAD, left atrial diameter; LV, left ventricular; LVMI, left ventricular mass index.
Table 3 Univariate regression analyses of factors predicting cardiovascular events

\begin{tabular}{|c|c|c|}
\hline Variable & r & $\mathrm{P}$-value \\
\hline Age & 0.170 & 0.031 \\
\hline Gender $(m=1, f=0)$ & 0.101 & 0.203 \\
\hline Body mass index & -0.150 & 0.057 \\
\hline eGFR & -0.284 & $<0.001$ \\
\hline Systolic blood pressure & 0.113 & 0.158 \\
\hline Diastolic blood pressure & 0.024 & 0.760 \\
\hline Heart rate & -0.060 & 0.461 \\
\hline Proteinuria (yes $=1$, no $=0$ ) & 0.191 & 0.015 \\
\hline Hemoglobin & -0.101 & 0.204 \\
\hline Serum albumin & -0.286 & $<0.001$ \\
\hline GNRI & -0.372 & $<0.001$ \\
\hline Hypertension (yes $=1$, no $=0$ ) & 0.130 & 0.100 \\
\hline Diabetes $($ yes $=1$, no $=0$ ) & 0.018 & 0.818 \\
\hline LV end-diastolic diameter & 0.091 & 0.091 \\
\hline LAD & 0.196 & 0.013 \\
\hline LV ejection fraction & -0.068 & 0.393 \\
\hline$E / e^{\prime}$ & 0.202 & 0.010 \\
\hline$e^{\prime}$ & -0.131 & 0.097 \\
\hline LVMI & 0.330 & $<0.001$ \\
\hline
\end{tabular}

Abbreviations: $e^{\prime}$, early diastolic mitral annulus motion; $E$, early mitral inflow; eGFR, estimated glomerular filtration rate; GNRI, geriatric nutritional risk index; LAD, left atrial diameter; LV, left ventricular; LVMI, left ventricular mass index. 
Table 4 Multivariate linear regression analyses of factors predicting cardiovascular events

\begin{tabular}{lrrr}
\hline Variable & B & \multicolumn{1}{c}{$\beta$} & P-value \\
\hline Age & 0.003 & 0.100 & 0.203 \\
Gender $(\mathrm{m}=1, \mathrm{f}=0)$ & 0.001 & 0.000 & 0.999 \\
eGFR & -0.003 & -0.166 & 0.033 \\
Hemoglobin & 0.003 & 0.016 & 0.868 \\
GNRI & -0.012 & -0.242 & 0.005 \\
Hypertension (yes =1, no=0) & 0.028 & 0.045 & 0.584 \\
Diabetes (yes =1, no=0) & -0.058 & -0.086 & 0.258 \\
LVMI & 0.002 & 0.241 & 0.004 \\
\hline
\end{tabular}

Abbreviations: eGFR, estimated glomerular filtration rate; GNRI, geriatric nutritional risk index; LVMI, left ventricular mass index.

Table 5 Hazards ratios for cardiovascular events according to Cox regression proportional hazards analysis

\begin{tabular}{lrccc}
\hline Variable & \multicolumn{1}{c}{$\beta$} & Hazard ratio & $95 \% \mathrm{Cl}$ & P-value \\
\hline Age & 0.114 & 1.121 & $1.013-1.241$ & 0.027 \\
Gender $(\mathrm{m}=1, \mathrm{f}=0)$ & -0.092 & 0.912 & $0.274-3.037$ & 0.881 \\
eGFR & -0.040 & 0.961 & $0.928-0.995$ & 0.024 \\
Hemoglobin & 0.248 & 1.282 & $0.843-1.950$ & 0.246 \\
GNRI & -0.121 & 0.886 & $0.807-0.973$ & 0.011 \\
Hypertension (yes =1, no=0) & 0.504 & 1.656 & $0.317-8.653$ & 0.550 \\
Diabetes (yes =1, no=0) & -0.660 & 0.517 & $0.145-1.840$ & 0.308 \\
LVMI & 0.017 & 1.017 & $1.002-1.033$ & 0.027
\end{tabular}

Abbreviations: $\beta$, standardized coefficients; $\mathrm{Cl}$, confidence interval; eGFR, estimated glomerular filtration rate; GNRI, geriatric nutritional risk index; LVMI, left ventricular mass index. for sex, hemoglobin levels and the prevalence of hypertension and diabetes (Table 5). These results suggest that malnutrition is an independent predictor of cardiovascular events, even in non-dialysis patients.

We then examined the impact of malnutrition, low eGFR and LVH on the occurrence of cardiovascular events using Kaplan-Meier curves. We stratified the data by the presence or absence of malnutrition, which was defined as GNRI $\leqslant 96$ based on our previous report. ${ }^{12}$ As shown in Figure 1, lower GNRI (Figure 1a), lower eGFR (Figure 1b) and the presence of LVH (Figure 1c) were significantly associated with higher rates of cardiovascular events. The presence of proteinuria (Figure 1d) and higher LAD (Figure 1e) were also associated with higher rates of cardiovascular events. The prevalence of hypertension was not significantly associated with cardiovascular events (Figure 1f).

We further examined the synergistic impact of malnutrition, low eGFR and LVH on cardiovascular events. The combination of lower eGFR and the presence of LVH was significantly associated with a higher rate of cardiovascular events $(P=0.003$; Figure 2a). The combination of lower eGFR and lower GNRI was also significantly associated with a higher rate of cardiovascular events $(P<0.001$; Figure 2b). Furthermore, the combination of LVH and lower GNRI was significantly associated with a higher rate of cardiovascular events not only in all patients $(P<0.001$; Figure 2c) but also in patients with CKD ( $n=46, P=0.014$; Supplementary Figure S1), suggesting that malnutrition, renal dysfunction and LVH have a synergistic impact on the incidence of cardiovascular events, even in non-dialysis patients.

Finally, we verified the possibility that proteinuria might decrease the serum level of albumin, which is determined by the formula for GNRI independent of malnutrition. However, there are no significant differences between the severity of dipstick proteinuria, serum a

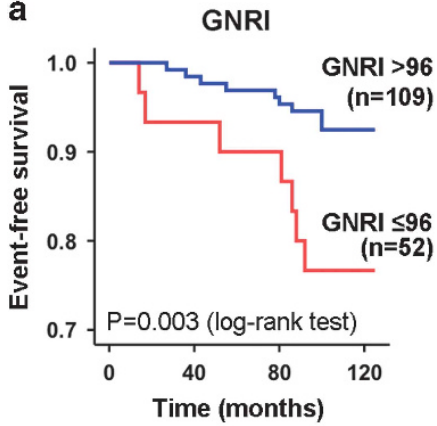

d

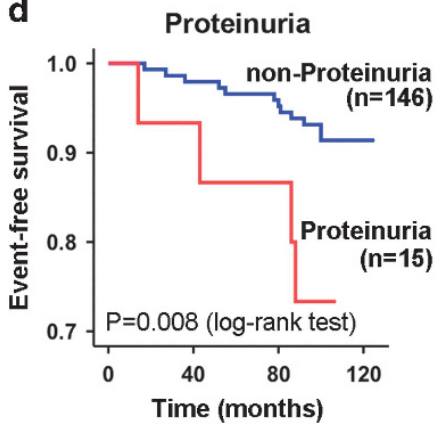

b

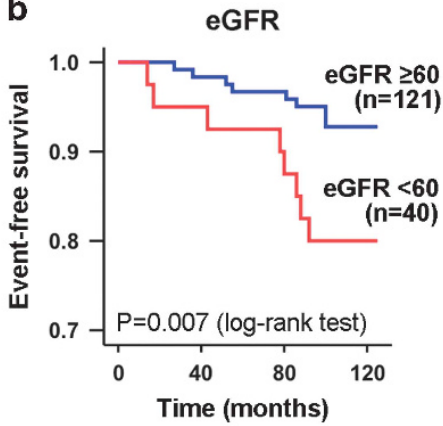

e

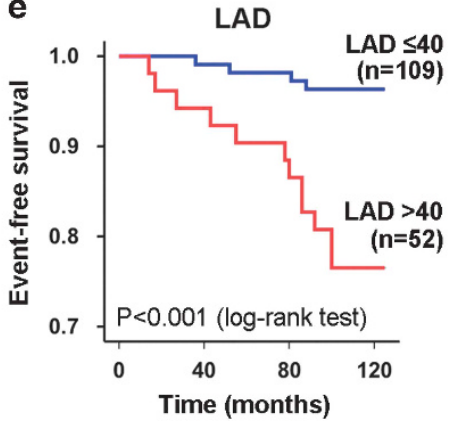

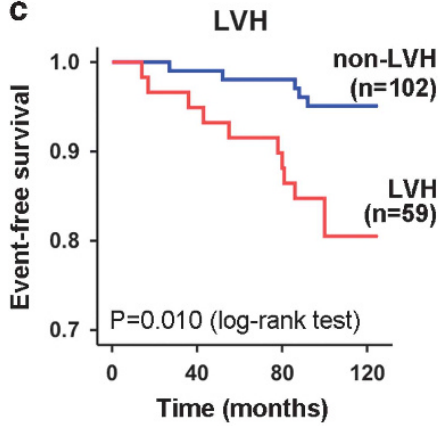

f

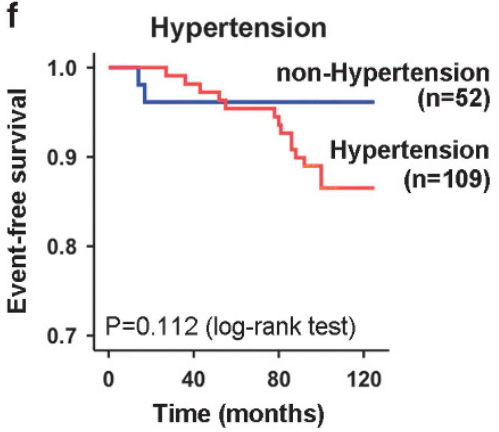

Figure 1 Kaplan-Meier curves for cardiovascular events according to (a) geriatric nutritional risk index (GNRI) $\leqslant 96$ or $>96$, (b) estimated glomerular filtration rate (eGFR) $<60$ or $\geqslant 60 \mathrm{ml} \mathrm{min-1}$ per $1.73 \mathrm{~m}^{2}$, (c) the presence or absence of left ventricular hypertrophy (LVH), (d) the presence or absence of proteinuria, (e) left atrial diameter (LAD) $<40$ or $\geqslant 40 \mathrm{~mm}$ and (f) the presence or absence of hypertension. Lower GNRI (a), lower eGFR (b), the presence of $\mathrm{LVH}(\mathbf{c})$, the presence of proteinuria (d) and higher LAD (e) were associated with a higher rate of cardiovascular events, but the prevalence of hypertension was not significantly associated with cardiovascular events (f). 


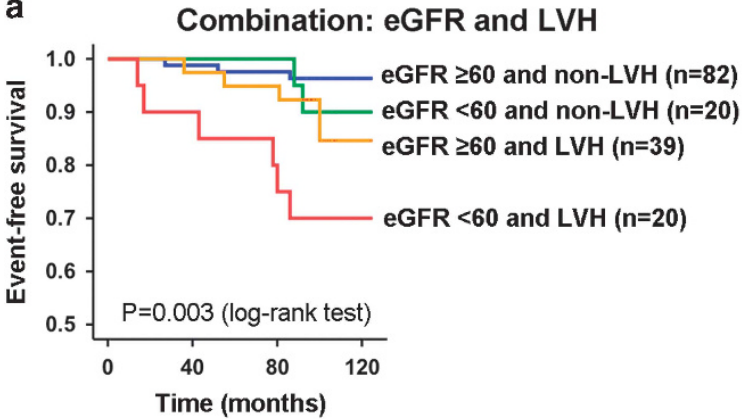

b Combination: eGFR and GNRI

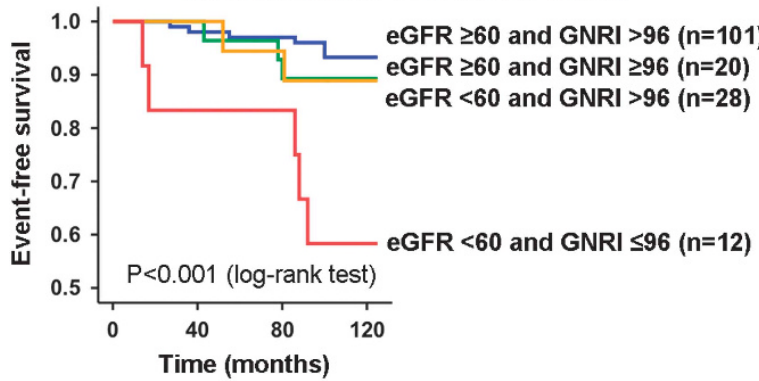

c Combination: LVH and GNRI

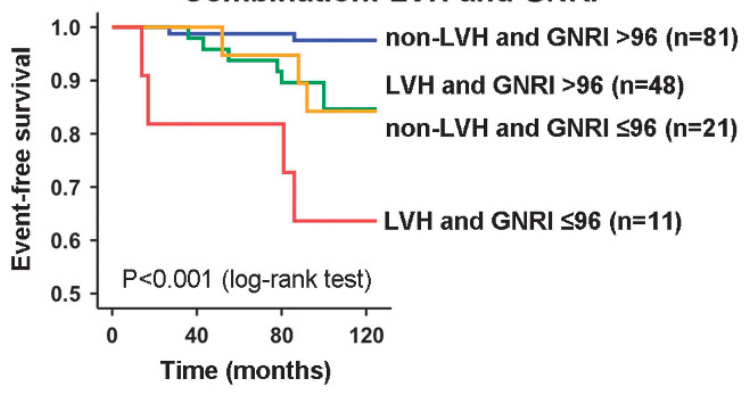

Figure 2 Kaplan-Meier curves for cardiovascular events according to (a) the combination of lower or higher estimated glomerular filtration rate (eGFR) ( $<60$ or $\geqslant 60 \mathrm{ml} \mathrm{min}-1$ per $1.73 \mathrm{~m}^{2}$, respectively) and the presence or absence of LVH, (b) the combination of lower or higher eGFR and lower or higher geriatric nutritional risk index (GNRI) $(\leqslant 96$ or $>96$, respectively) or (c) the combination of the presence or absence of LVH and lower or higher GNRI. albumin (Figure 3a) and GNRI (Figure 3b), suggesting that proteinuria did not affect nutritional status in this study.

\section{DISCUSSION}

To our knowledge, this study is the first to describe the following: (1) malnutrition, low eGFR and LVH are all independent predictors of cardiovascular events and exhibit a synergistic impact on cardiovascular events in non-dialysis patients who were followed up for $>7$ years; and (2) a cutoff value of GNRI of $\leqslant 96$ is useful for predicting cardiovascular events, even in non-dialysis patients.

This study demonstrated that eGFR was significantly and positively associated with GNRI, which is useful for predicting cardiovascular events. GNRI was originally used to predict malnutrition-related complications and mortality in elderly hospitalized patients, and the defined cutoff values according to weight loss and albumin concentrations were: GNRI $<82$, major nutrition-related risk; GNRI 82 to $<92$, moderate nutrition-related risk; GNRI 92 to $\leqslant 98$, low nutritionrelated risk; GNRI >98, no risk. ${ }^{9}$ Several studies in hemodialysis patients have reported that the GNRI cutoff value is $\sim 90$ to $91 .^{10}$ Additionally, GNRI was also reported to be involved in predicting death due to cardiovascular events or the rehospitalization in patients with chronic heart failure. ${ }^{18,19}$ However, we have recently reported that the cutoff value of GNRI for predicting all-cause and cardiovascular mortality is 96 in hemodialysis patients. ${ }^{12}$ In this study, we clearly showed that a cutoff value of GNRI of $\leqslant 96$ can be used in predicting cardiovascular events, even in non-dialysis patients.

GNRI uses body weight to assess nutritional status. Body weight is affected by fluid status, which may lead to an underestimation of nutritional status in patients with CKD or chronic heart failure. In this study, however, we demonstrated that GNRI was not associated with LAD, LVMI or LV end-diastolic diameter, suggesting that GNRI was an independent predictor of cardiovascular events regardless of volume overload. Although proteinuria might decrease the serum level of albumin, which contributes to the calculation of GNRI, our results demonstrate that the levels of proteinuria were not associated with serum albumin and GNRI; these results suggest that proteinuria did not affect nutritional status in this study. However, the use of diuretics at baseline was significantly higher in patients who developed cardiovascular events in this study. Several studies have revealed that loop diuretic use is associated with increased mortality risk in chronic heart failure patients because it increases the activity of the reninangiotensin-aldosterone system. ${ }^{20}$ Diuretics may also induce
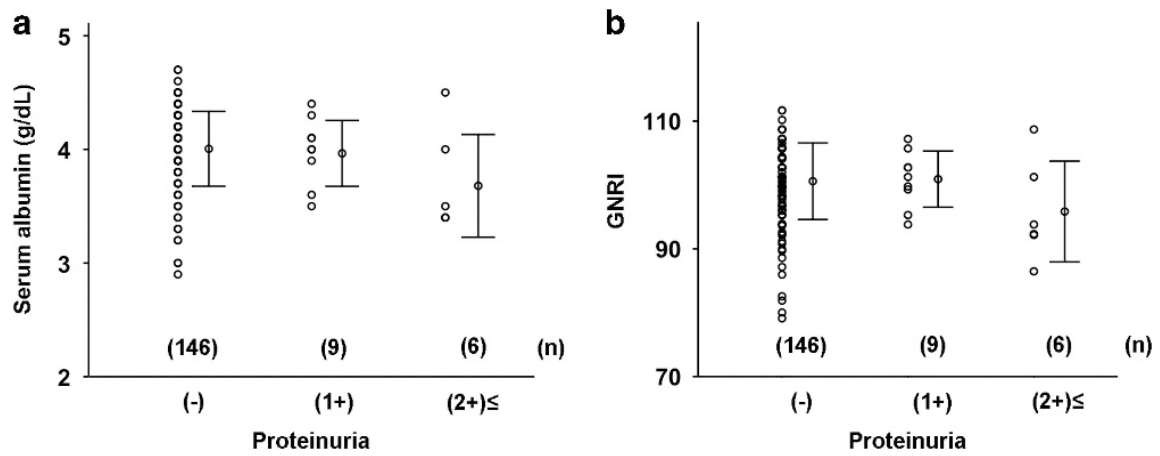

Figure 3 Scatter dot plots of the levels of serum albumin (a) and geriatric nutritional risk index (GNRI) (b) according to the severity of dipstick proteinuria. Error bars represent the mean \pm s.d. for each group. There are no significant differences between each group either in serum albumin (a) or GNRI (b). 
hypokalemia, ${ }^{21}$ thus promoting cardiac arrhythmias and sudden death.

Our subanalysis of the effects of LVH and GNRI on cardiovascular events in patients with CKD revealed that the combination of LVH and lower GNRI was significantly associated with a higher rate of cardiovascular events. Age, systolic blood pressure, LVM and left atrial size have been reported to contribute to the incidence of atrial fibrillation. ${ }^{22,23}$ These factors might act as mediators between CKD and atrial fibrillation as reported in several previous studies. ${ }^{24,25}$ Because GFR generally decreases with age, pressure and volume load augmented by renal dysfunction directly increase arterial stiffness, LVM and left atrial size. ${ }^{26,27}$ Recent studies indicate that subclinical nephrosclerosis is linked to LVH independent of classical atherogenic factors. ${ }^{28}$ Furthermore, a strong correlation exists among three separate clinical entities of malnutrition, inflammation and atherosclerosis, which coexist in patients with end-stage renal disease and are collectively known as the malnutrition-inflammation-atherosclerosis syndrome. ${ }^{7}$ This correlation suggests that chronic inflammation might also affect cardiovascular events in this study.

Our study has several limitations. First, it is a retrospective observational study with a relatively small sample size derived from a single treatment center; thus, it is open to disadvantages of such a study design including selection bias. Second, we did not monitor blood pressure during the observational period. Although baseline blood pressures were not associated with cardiovascular events in our study, we cannot discount the impact of hypertension, which is a major risk factor for cardiovascular events. Third, we did not evaluate the prevalence of atrial fibrillation or smoking, and we were unable to evaluate an interaction between these factors and the incidence of cardiovascular events. Larger prospective studies are needed to determine the synergistic impact of malnutrition and LVH in patients with CKD.

In conclusion, this study clearly demonstrated that malnutrition, low eGFR and LVH are independent determinants of the occurrence of cardiovascular events, and they synergistically increase the rates of these events in the long term. The evaluation and management of nutritional status in patients with LVH is critical in preventing cardiovascular complications even in non-dialysis patients.

\section{CONFLICT OF INTEREST}

KM has received a travel grant from the Hokkaido Heart Association. The remaining authors declare no conflict of interest.

\section{ACKNOWLEDGEMENTS}

This study was supported, in part, by a Grant-in-Aid for Progressive Renal Diseases Research, Research on Rare and Intractable Disease, from the Ministry of Health, Labor and Welfare of Japan. We thank the Asahikawa Medical University Hospital staff for their help with data collection.

1 Meguid El Nahas A, Bello AK. Chronic kidney disease: the global challenge. Lancet 2005; 365: 331-340.

2 Hamaguchi S, Tsuchihashi-Makaya M, Kinugawa S, Yokota T, Ide T, Takeshita A, Tsutsui H. Chronic kidney disease as an independent risk for long-term adverse outcomes in patients hospitalized with heart failure in Japan. Report from the Japanese Cardiac Registry of Heart Failure in Cardiology (JCARE-CARD). Circ J 2009; 73: 1442-1447.

3 Salvetti M, Muiesan ML, Paini A, Monteduro C, Bonzi B, Galbassini G, Belotti E, Movilli E, Cancarini G, Agabiti-Rosei E. Myocardial ultrasound tissue characterization in patients with chronic renal failure. J Am Soc Nephrol 2007; 18: 1953-1958.
4 Yoshitomi R, Fukui A, Nakayama M, Ura Y, Ikeda H, Oniki H, Tsuchihashi T, Tsuruya K, Kitazono T. Sex differences in the association between serum uric acid levels and cardiac hypertrophy in patients with chronic kidney disease. Hypertens Res 2014; 37: 246-252.

5 McMahon LP, Roger SD, Levin A. Development, prevention, and potential reversal of left ventricular hypertrophy in chronic kidney disease. J Am Soc Nephrol 2004; 15: 1640-1647.

6 Glassock RJ, Pecoits-Filho R, Barberato SH. Left ventricular mass in chronic kidney disease and ESRD. Clin J Am Soc Nephrol 2009; 4 (Suppl 1):S79-S91.

7 Pecoits-Filho R, Lindholm B, Stenvinkel P. The malnutrition, inflammation, and atherosclerosis (MIA) syndrome - the heart of the matter. Nephrol Dial Transplant 2002; 17 (Suppl 11):28-31.

8 Amparo FC, Kamimura MA, Molnar MZ, Cuppari L, Lindholm B, Amodeo C, Carrero JJ, Cordeiro AC. Diagnostic validation and prognostic significance of the MalnutritionInflammation Score in nondialyzed chronic kidney disease patients. Nephrol Dial Transplant 2015; 30: 821-828.

9 Bouillanne O, Morineau G, Dupont C, Coulombel I, Vincent JP, Nicolis I, Benazeth S, Cynober L, Aussel C. Geriatric Nutritional Risk Index: a new index for evaluating at-risk elderly medical patients. Am J Clin Nutr 2005; 82: 777-783.

10 Yamada K, Furuya R, Takita T, Maruyama Y, Yamaguchi Y, Ohkawa S, Kumagai H. Simplified nutritional screening tools for patients on maintenance hemodialysis. Am J Clin Nutr 2008; 87: 106-113.

11 Kobayashi I, Ishimura E, Kato Y, Okuno S, Yamamoto T, Yamakawa T, Mori K, Inaba M, Nishizawa Y. Geriatric Nutritional Risk Index, a simplified nutritional screening index, is a significant predictor of mortality in chronic dialysis patients. Nephrol Dial Transplant 2010; 25: 3361-3365.

12 Nakagawa N, Matsuki M, Yao N, Hirayama T, Ishida H, Kikuchi K, Hasebe N. Impact of metabolic disturbances and malnutrition-inflammation on 6-year mortality in Japanese patients undergoing hemodialysis. Ther Apher Dial 2015; 19 : 30-39.

13 Matsuzawa Y, Nakamura T, Takahashi M, Ryo M, Inoue S, Ikeda Y, Ohno M, Sakata T, Fukagawa K, Saitoh Y, Sato Y, Shirai K, Miyazaki S, Tokunaga K, Yamanouchi K, Shibasaki T, Nagai M, Japan Society Study O. New criteria for 'obesity disease' in Japan. Circ J 2002; 66: 987-992.

14 Lang RM, Bierig M, Devereux RB, Flachskampf FA, Foster E, Pellikka PA, Picard MH, Roman MJ, Seward J, Shanewise JS, Solomon SD, Spencer KT, Sutton MS, Stewart WJ. Recommendations for chamber quantification: a report from the American Society of Echocardiography's Guidelines and Standards Committee and the Chamber Quantification Writing Group, developed in conjunction with the European Association of Echocardiography, a branch of the European Society of Cardiology. J Am Soc Echocardiogr 2005; 18 : 1440-1463.

15 Mancia G, De Backer G, Dominiczak A, Cifkova R, Fagard R, Germano G, Grassi G, Heagerty AM, Kjeldsen SE, Laurent S, Narkiewicz K, Ruilope L, Rynkiewicz A, Schmieder RE, Boudier HA, Zanchetti A. 2007 ESH-ESC Practice Guidelines for the Management of Arterial Hypertension: ESH-ESC Task Force on the Management of Arterial Hypertension. J Hypertens 2007; 25: 1751-1762.

16 Ommen SR, Nishimura RA, Appleton CP, Miller FA, Oh JK, Redfield MM, Tajik AJ. Clinical utility of Doppler echocardiography and tissue Doppler imaging in the estimation of left ventricular filling pressures: a comparative simultaneous Dopplercatheterization study. Circulation 2000; 102: 1788-1794.

17 Sohn DW, Kim YJ, Park YB, Choi YS. Clinical validity of measuring time difference between onset of mitral inflow and onset of early diastolic mitral annulus velocity in the evaluation of left ventricular diastolic function. J Am Coll Cardiol 2004; 43: 2097-2101.

18 Kinugasa Y, Kato M, Sugihara S, Hirai M, Yamada K, Yanagihara K, Yamamoto K. Geriatric nutritional risk index predicts functional dependency and mortality in patients with heart failure with preserved ejection fraction. Circ J 2013; 77: 705-711.

19 Izawa KP, Watanabe S, Hirano Y, Yamamoto S, Oka K, Suzuki N, Kida K, Suzuki K, Osada N, Omiya K, Brubaker PH, Shimizu H, Akashi YJ. The relation between Geriatric Nutritional Risk Index and muscle mass, muscle strength, and exercise capacity in chronic heart failure patients. Int J Cardiol 2014; 177: 1140-1141.

20 Bayliss J, Norell M, Canepa-Anson R, Sutton G, Poole-Wilson P. Untreated heart failure: clinical and neuroendocrine effects of introducing diuretics. Br Heart J 1987; 57: 17-22.

21 Salerno DM, Asinger RW, Elsperger J, Ruiz E, Hodges M. Frequency of hypokalemia after successfully resuscitated out-of-hospital cardiac arrest compared with that in transmural acute myocardial infarction. Am J Cardiol 1987; 59: 84-88.

22 Prystowsky EN, Benson DW, Fuster V, Hart RG, Kay GN, Myerburg RJ, Naccarelli GV, Wyse DG. Management of patients with atrial fibrillation-a statement for healthcare professionals from the subcommittee on electrocardiography and electrophysiology, American Heart Association. Circulation 1996; 93: 1262-1277.

23 Allessie MA, Boyden PA, Camm AJ, Kleber AG, Lab MJ, Legato MJ, Rosen MR, Schwartz PJ, Spooner PM, Van Wagoner DR, Waldo AL. Pathophysiology and prevention of atrial fibrillation. Circulation 2001; 103: 769-777.

24 Tanabe Y, Kawamura Y, Sakamoto N, Sato N, Kikuchi K, Hasebe N. Blood pressure control and the reduction of left atrial overload is essential for controlling atrial fibrillation. Int Heart J 2009; 50: 445-456. 
25 Chinda J, Nakagawa N, Kabara M, Matsuki M, Endo H, Saito T, Sawada J, Katayama T, Sato N, Hasebe N. Impact of decreased estimated glomerular filtration rate on Japanese acute stroke and its subtype. Intern Med 2012; 51: 1661-1666.

26 Nakagawa N, Takahashi F, Chinda J, Kobayashi M, Hayashi Y, Abe M, Saijo Y, Kikuchi K, Hasebe N. A newly estimated glomerular filtration rate is independently associated with arterial stiffness in Japanese patients. Hypertens Res 2008; 31 193-201.
27 Talib AA, Nakagawa N, Saito E, Matsuki M, Kobayashi M, Akasaka K, Hirayama T, Ishida $\mathrm{H}$, Sato $\mathrm{N}$, Hasebe $\mathrm{N}$. The balance of fetuin-A and osteoprotegerin is independently associated with diastolic dysfunction in hemodialysis patients. Hypertens Res 2012; 35: 426-433.

28 Haruyama N, Tsuchimoto A, Masutani K, Nagata M, Kitada H, Tanaka M, Tsuruya K, Kitazono T. Subclinical nephrosclerosis is linked to left ventricular hypertrophy independent of classical atherogenic factors. Hypertens Res 2014; 37: 472-477.

Supplementary Information accompanies the paper on Hypertension Research website (http://www.nature.com/hr) 